\title{
Co-administration of a Tumor-Penetrating Peptide Enhances the Efficacy of Cancer Drugs
}

\author{
Kazuki N. Sugahara $1,{ }^{*}$, Tambet Teesalu $1,{ }^{*}$, Priya Prakash Karmali ${ }^{2}$, Venkata Ramana \\ Kotamraju ${ }^{1}$, Lilach Agemy ${ }^{1}$, Daniel R. Greenwald ${ }^{3}$, and Erkki Ruoslahti ${ }^{1,2, \dagger}$ \\ ${ }^{1}$ Vascular Mapping Laboratory, Center for Nanomedicine, Sanford-Burnham Medical Research \\ Institute at UCSB, Biology II Bldg., University of California, Santa Barbara, CA 93106-9610 \\ ${ }^{2}$ Cancer Research Center, Sanford-Burnham Medical Research Institute, 10901 N. Torrey Pines \\ Rd., La Jolla, CA 92037 \\ ${ }^{3}$ Santa Barbara Hematology Oncology Medical Group, Cancer Center of Santa Barbara, 540 \\ West Pueblo St., Santa Barbara, CA 93105, U.S.A.
}

\begin{abstract}
Poor penetration of anti-cancer drugs into tumors can be an important factor limiting their efficacy. Studying mouse tumor models, we show that a previously characterized tumorpenetrating peptide, iRGD (CRGDK/RGPD/EC), increased vascular and tissue permeability in a tumor-specific and neuropilin-1-dependent manner, allowing co-administered drugs to penetrate into extravascular tumor tissue. Importantly, this effect did not require the drugs to be chemically conjugated to the peptide. Systemic injection with iRGD improved the therapeutic index of drugs of various compositions including a small molecule (doxorubicin), nanoparticles (nab-paclitaxel and doxorubicin liposomes), and a monoclonal antibody (trastuzumab). Thus, co-administration of iRGD may be a valuable way to enhance the efficacy of anti-cancer drugs while reducing their side effects, a primary goal of cancer therapy research.
\end{abstract}

The therapeutic efficacy of many anti-cancer drugs is limited by their poor penetration into tumor tissue and by their adverse effects on healthy cells, which limits the dose of drug that can be safely administered to cancer patients. In solid tumors, many anti-cancer drugs penetrate only 3-5 cell diameters from the blood vessels, leading to reduced efficacy and the development of drug resistance $(1,2)$. We recently identified a tumor-penetrating peptide, iRGD (CRGDK/RGPD/EC), that when chemically conjugated to a drug can carry the drug deep into extravascular tumor tissue (3). Like conventional RGD peptides, iRGD homes to tumors by initially binding to $\alpha \mathrm{v}$ integrins that are specifically expressed on the endothelium of tumor vessels $(3,4,5)$. iRGD is then proteolytically cleaved in the tumor to produce CRGDK/R. The truncated peptide loses much of its integrin-binding activity, but gains affinity for neuropilin-1 (NRP-1) because of the C-terminal exposure of a conditional C-end Rule (CendR) motif (R/KXXR/K) (6). The NRP-1 binding triggers tissue penetration, which is tumor specific because the cleavage requires prior binding of the peptide to integrins.

\footnotetext{
${ }^{\dagger}$ To whom correspondence should be addressed. ruoslahti@burnham.org.

*These authors contributed equally to this work.

Supporting Online Material

www.sciencemag.org

Materials and Methods

Figs. S1 to S16

Table S1

References
} 
These features confer on iRGD a tumor-specific tissue penetration activity. Here we have explored whether the iRGD peptide can enhance cancer drug delivery and activity when it is administered as a combination therapy with drugs that are not chemically conjugated to it. This would be advantageous because already approved drugs could be used without creating a new chemical entity, and because coupling often interferes with drug activity

The activity of peptides and proteins that bind to NRP-1 through a C-terminal CendR motif can be demonstrated by monitoring vascular permeability (6-8). We examined whether the CendR-triggered vascular permeability may play a role in the iRGD tumor penetrating activities using human tumor xenografts growing in immunodeficient mice and in de novo mouse tumors growing in transgenic mice. Indeed, chemically synthesized iRGD peptide, when co-injected with the albumin-binding dye Evans Blue (9-11), caused tumor-specific accumulation of the dye in all 5 tumor models we tested ( 1 breast and 2 prostate cancers, and 2 pancreatic adenocarcinomas), including secondary invasion sites and disseminated tumors (fig. S1 A and fig. S2, A and B). The accumulation of the dye was dependent on the dose of iRGD administered, and peaked at about $400 \%$ over the dye alone (fig. S1B). NonCendR RGD peptides, RGD-4C (CDCRGDCFC) (12) and cyclo(-RGDfK-) (13), and a scrambled iRGD with no CendR motif (CRGDDGPKC) did not increase the permeability (fig. S1C and fig. S2C). Pre-injection of an anti-NRP-1 antibody inhibited the iRGDinduced permeability (fig. S1D). CRGDK, the truncated iRGD peptide with an exposed CendR motif, enhanced in a dose-dependent manner local vascular permeability in the skin (fig. S3) (6). When injected systemically, CRGDK increased permeability in the tumors as well as in the lungs and heart. CRGDK was somewhat selective for the tumors, likely because of its residual affinity to integrins and the generally high expression of NRP-1 in tumors (fig. S1C) $(3,6,14)$.

The tumor-specific increase in tissue access mediated by iRGD suggested a way of improving the delivery of compounds to tumor parenchyma (fig. S4). A fluorescein-labeled non-CendR peptide CRGDC (FAM-CRGDC; $1.3 \mathrm{kDa}$ ), which minimally penetrates tumors by itself $(3,15)$, showed enhanced extravascular distribution upon iRGD co-injection (fig. S5A). The co-injection caused a 300\% increase in both the FAM-CRGDC-positive areas (fig. S5B) and spreading of FAM-CRGDC (fig. S5C) in the tumors. Similar results were obtained with $3 \mathrm{kDa}$ and $10 \mathrm{kDa}$ dextrans (fig. S5). Two nanoparticles, iron-oxide nanoworms (16) and T7 phage, also extravasated and showed enhanced accumulation in the tumor upon iRGD co-injection (fig. S6). These results show that iRGD can increase the tumor accumulation of compounds with different sizes and chemical properties.

Intravenously injected molecules and nanoparticles slowly extravasate into perivascular areas in tumor tissue through passive leakage $(17,18)$. The rapid and deep tumor penetration of the probes co-injected with iRGD prompted us to study the possibility that CendR might be triggering an active transport process. To exclude circulatory effects, we used fresh explants of PPC1 human prostate tumors in this study. Phage particles expressing the iRGD peptide on their surface (3), but not inert control phage, penetrated into PPC-1 prostate cancer xenograft explants, advancing about $4 \mathrm{~mm}$ in 90 minutes (fig. S7). The penetration was inhibited by anti-NRP-1, sodium azide, and by lowering the temperature, suggesting that the penetration process was energy-dependent. Importantly, inert phage penetrated into the explants in the presence of iRGD, but not with a control peptide.

To study the effect of iRGD on drug delivery and efficacy, we administered the peptide as a combination therapy with several types of cancer drugs to mice bearing human tumor xenografts. We first compared the delivery efficiency of this combination regimen to that of the cancer drug alone and to that of the cancer drug when it was chemically conjugated to the peptide. Nab-paclitaxel (Abraxane; ABX), a $130 \mathrm{~nm}$ nanoparticle consisting of albumin- 
embedded paclitaxel $(3,19)$, when intravenously injected with iRGD, accumulated 12 fold more in orthotopic BT474 human breast tumors than ABX given alone and penetrated far from the tumor vessels (Fig. 1A and fig. S8). Daily treatment of mice with the ABX/iRGD combination inhibited the growth of BT474 tumors, which were resistant to equivalent doses of ABX alone (Fig. 1B). The combination was slightly more effective than the conjugated drug in inhibiting tumor growth, as well as in tumor accumulation, but the differences did not reach statistical significance. Similar results were obtained in orthotopic 22Rv1 human prostate tumors (Fig. 1, C and D).

We next used another clinically relevant system to evaluate the effect of the combination therapy on the therapeutic index, the ratio of the dose taken to elicit a therapeutic effect and the lethal dose. Doxorubicin (DOX) and DOX liposomes have clinical activity in a number of tumor types including prostate cancer (20). We treated orthotopic 22Rv1 tumors with a combination of iRGD and DOX $(0.6 \mathrm{kDa})$. Free DOX co-injected with iRGD penetrated the tumors and accumulated 7 fold more in the tumors than DOX given alone (Fig. 2, A and B). The combination therapy that included $1 \mathrm{mg} / \mathrm{kg}$ DOX was as potent as $3 \mathrm{mg} / \mathrm{kg}$ DOX alone, a dose that reaches the cumulative maximum tolerated dose (MTD) (Fig. 3C) (21).

Combining iRGD with $3 \mathrm{mg} / \mathrm{kg}$ of DOX potentiated the activity of DOX, inducing nearly complete tumor growth inhibition (Fig. 2C). Interestingly, tumors from both the 1 and $3 \mathrm{mg} /$ $\mathrm{kg}$ DOX groups combined with iRGD showed stronger TUNEL staining, an indicator of cell death, than tumors treated with $3 \mathrm{mg} / \mathrm{kg}$ DOX alone (Fig. 2D). Cardiotoxicity, the main dose-limiting toxicity of DOX, is manifested as cardiomyocyte apoptosis (22). The iRGD combination had the same cardiotoxicity as an equivalent dose of DOX alone (Fig. 2D). The body weight shift was also the same whether DOX was given alone or combined with iRGD (fig. S9). Similar results were obtained with orthotopic 4T1 mouse breast tumors (fig. S9 and fig. S10). iRGD alone at the dose used in the combination group showed no effect on tumor growth (Fig. 2C), supporting the notion that the effects of the combination are due to improved drug penetration.

We also tested DOX in a liposomal formulation (particle diameter $120 \mathrm{~nm}$ ). The results were similar to those with free DOX with regard to treatment efficacy (Fig. 3A), tumor accumulation of the drug (14 fold; Fig. 3B), tumor penetration (fig. S11), and toxicity (Fig. $3 \mathrm{C}$ and fig. S9). In addition, we tested a non-CendR RGD in this system and found that it did not enhance the efficacy of the drug (Fig. 3A). These results indicate that in comparison to drug administered alone, the iRGD combination therapy provides equivalent or better anti-tumor efficacy at a 3 -fold lower dose of the drug, with a commensurate reduction in toxicity. They also show that this therapy regimen can be effective with drugs ranging in size from a small molecular weight chemical (DOX) to nanoparticles (ABX, DOX liposomes).

Finally, we tested the iRGD-combination regimen in mice bearing tumors derived from BT474 human breast tumor cells, which overexpress HER2/neu/ErbB2. This growth factor receptor is the target of trastuzumab, a therapeutic monoclonal antibody in clinical use for the treatment of breast cancer $(23,24)$. Co-injection of iRGD resulted in a 14-fold increase in trastuzumab-positive areas within the tumor (Fig. 4A). ELISA showed that iRGD enhanced the accumulation of trastuzumab in the tumors 40 fold; this increase is likely due to enhanced access and binding of the antibody to the tumor cells (Fig. 4B). Combining trastuzumab with iRGD increased the drug potency at different trastuzumab dose levels (Fig. 4C). At a clinical dose $(9 \mathrm{mg} / \mathrm{kg})$, the combination eradicated all tumors in the mice, whereas the equivalent dose of trastuzumab alone only slowed down tumor growth (Fig. 4C). The eradicated tumors did not relapse during a 2-week observation period after the treatment was stopped. 
The iRGD-mediated enhancement in drug penetration persisted even after more than 3 weeks of antibody treatment (fig. S12 and fig. S13 for DOX and trastuzumab, respectively). However, the difference between the combination and the drug alone was smaller than with single dosing (compare fig. S13B with Fig. 4A). This explains the lack of linear relationship between the single dosing of the drugs, which gave 7-40 fold more drug accumulation, and the long-term treatments, which gave 3 fold stronger anti-tumor effects.

We also tested the possibility that iRGD might have the adverse effect of enhancing metastasis by promoting intravasation of tumor cells. No macroscopic metastases were found in mice treated with iRGD. Immunofluorescence for human leukocyte antigens (HLAs) and quantitative PCR measuring human genomic DNA revealed no human cells in organs collected at the end of the treatments and the low levels of DNA were similar in the iRGD and control groups in the BT474 and 22Rv1 models (fig. S14 to fig. S16) $(25,26)$.

The CendR penetration effect of the tumor targeting methodology described here is distinct from the Enhanced Permeability and Retention (EPR) effect: (i) EPR is a passive leakage from tumor vessels $(17,18)$, whereas the CendR penetration is receptor-mediated and energy-dependent, (ii) CendR is more effective than the EPR (e.g. fig. S5), (iii) CendR causes extravasation within minutes, whereas EPR requires 6-8 hours to peak $(3,17)$, and (iv) CendR is effective with small molecules, whereas EPR is not (17). However, there may be a CendR component in EPR because VEGF-165, which is involved in the process, has an active CendR motif $(6,7,17)$.

Administration of the tumor-penetrating peptide as a combination therapy bypasses two limitations of strategies in which the peptide is conjugated to the drug and delivered as a single agent: (i) The combination therapy does not require drug modification, which may impair drug activity, and (ii) The capacity of the system is not exceeded as easily as that of the conjugate delivery. While delivery of the conjugated drug depends on a finite number of target receptors, delivery of the peptide and drug as separate entities triggers a bulk transfer of the bystander drugs into the tumor tissue (27).

Attempts have been made to permeabilize tumor vasculature with VEGF and bradykinin for enhanced access of blood-borne molecules to tumor interstitium $(28,29)$, but the tumor specificity has been limited. One possible advantage of iRGD is that systemic administration of this peptide results in a tissue penetration effect that appears to be selective for tumors.

We observed enhanced tumor-specific delivery of the 10 different compounds we tested (from a $0.6 \mathrm{kDa}$ molecule up to a $130 \mathrm{~nm}$ particle), suggesting that the iRGD peptide might help improve the performance of a wide range of cancer drugs or tumor imaging agents. Conceivably, the system can be further improved -- for example, by employing multimeric iRGD, structurally stabilized iRGD, other tumor-homing CendR peptides (30-33), or peptide combinations, or by optimization of the dosing and administration schedules. Moreover, the efficacy and the specificity of the iRGD-mediated delivery remain to be shown in human patients. Human tumor vessels and tumor cells express the relevant $\alpha v$ integrins $(4,5)$ and NRP-1 (14), and iRGD and its fragment bind to them (3), suggesting that the system will translate to the treatment of human cancer. Increasing the therapeutic index of standard therapy may enhance efficacy while reducing toxicity, the primary goal of modern cancer therapy.

\section{Supplementary Material}

Refer to Web version on PubMed Central for supplementary material. 


\section{Acknowledgments}

We thank D. Hanahan for transgenic mice, R. M. Hoffman for the GFP-PC-3 cell line, M. J. Sailor and J-H Park for advice on the synthesis of iron oxide nanoworms, and E. Engvall for comments on the manuscript. This work was supported by grants CA104898, CA 119414, CA 119335, CA124427, CA115410, and 30199 from the National Cancer Institute of the NIH and grants BC076050 and BC08544 from the Department of Defense. The authors and their institutions have patents on methods and compositions related to peptides and proteins with C-terminal elements and related to internalizing RGD peptides.

\section{References and Notes}

1. Hambley TW, Hait WN. Cancer Res 2009;69:1259. [PubMed: 19208831]

2. Minchinton AI, Tannock IF. Nat Rev Cancer 2006;6:583. [PubMed: 16862189]

3. Sugahara KN, et al. Cancer Cell 2009;16:510. [PubMed: 19962669]

4. Ruoslahti E. Nat Rev Cancer 2002;2:83. [PubMed: 12635171]

5. Desgrosellier JS, Cheresh DA. Nat Rev Cancer 2010;10:9. [PubMed: 20029421]

6. Teesalu T, Sugahara KN, Kotamraju VR, Ruoslahti E. Proc Natl Acad Sci USA 2009;106:16157. [PubMed: 19805273]

7. Jia H, et al. J Biol Chem 2006;281:13493. [PubMed: 16513643]

8. Acevedo LM, Barillas S, Weis SM, Gothert JR, Cheresh DA. Blood 2008;111:2674. [PubMed: 18180379]

9. See supporting material on Science Online for methods and additional data. Statistical analyses are summarized in table $\mathrm{S} 1$.

10. Miles AA, Miles EM. J Physiol 1952;118:228. [PubMed: 13000707]

11. Murohara T, et al. Circulation 1998;97:99. [PubMed: 9443437]

12. Koivunen E, Wang B, Ruoslahti E. Biotechnology (N Y) 1995;13:265. [PubMed: 9634769]

13. Murphy EA, et al. Proc Natl Acad Sci USA 2008;105:9343. [PubMed: 18607000]

14. Pellet-Many C, Frankel P, Jia H, Zachary I. Biochem J 2008;411:211. [PubMed: 18363553]

15. Koivunen E, Gay DA, Ruoslahti E. J Biol Chem 1993;268:20205. [PubMed: 7690752]

16. Park JH, et al. Small 2009;5:694. [PubMed: 19263431]

17. Maeda H, Fang J, Inutsuka T, Kitamoto Y. Int Immunopharmacol 2003;3:319. [PubMed: 12639809]

18. Yuan F, et al. Cancer Res 1995;55:3752. [PubMed: 7641188]

19. Karmali PP, et al. Nanomedicine 2009;5:73. [PubMed: 18829396]

20. Petrioli R, Fiaschi AI, Francini E, Pascussi A, Francini G. Cancer Treat Rev 2008;34:710. [PubMed: 18620815]

21. Pastorino F, et al. Clin Cancer Res 2008;14:7320. [PubMed: 19010847]

22. Arola OJ, et al. Cancer Res 2000;60:1789. [PubMed: 10766158]

23. Fendly BM, et al. Cancer Res 1990;50:1550. [PubMed: 1689212]

24. Carter P, et al. Proc Natl Acad Sci USA 1992;89:4285. [PubMed: 1350088]

25. Primeau T, et al. Immunol Res 2005;32:109. [PubMed: 16106063]

26. Tagle DA, Collins FS. Hum Mol Genet 1992;1:121. [PubMed: 1301148]

27. Ruoslahti E, Bhatia SN, Sailor MJ. J Cell Biol. Mar 15;2010 Epub ahead of print.

28. Monsky WL, et al. Cancer Res 1999;59:4129. [PubMed: 10463618]

29. Black KL, Ningaraj NS. Cancer Control 2004;11:165. [PubMed: 15153840]

30. Hoffman JA, et al. Cancer Cell 2003;4:383. [PubMed: 14667505]

31. Joyce JA, et al. Cancer Cell 2003;4:393. [PubMed: 14667506]

32. Laakkonen P, Porkka K, Hoffman JA, Ruoslahti E. Nat Med 2002;8:751. [PubMed: 12053175]

33. Porkka K, Laakkonen P, Hoffman JA, Bernasconi M, Ruoslahti E. Proc Natl Acad Sci USA 2002;99:7444. [PubMed: 12032302] 
A

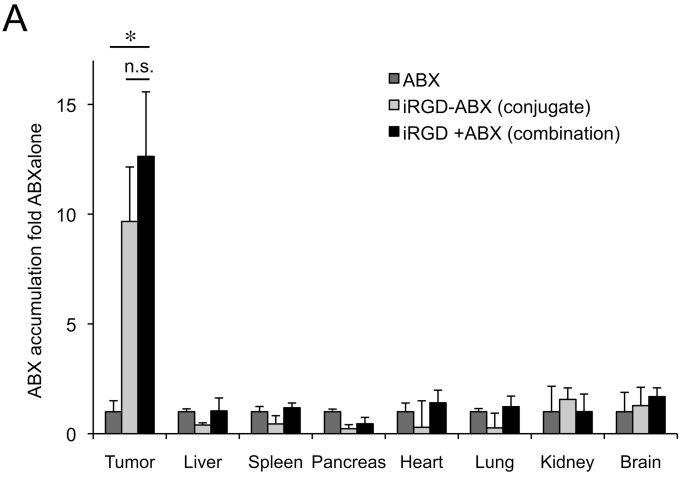

C

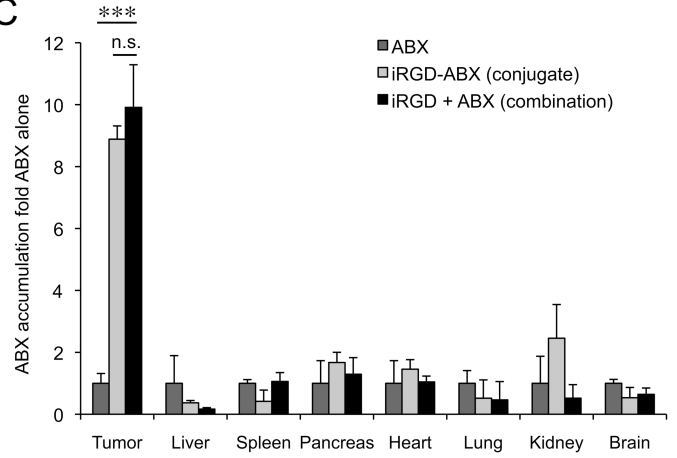

B

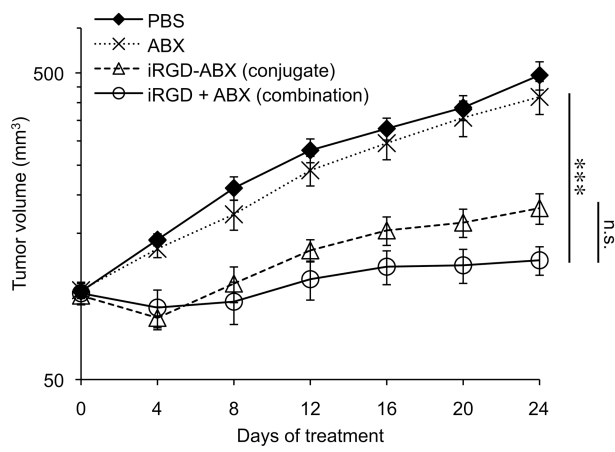

D

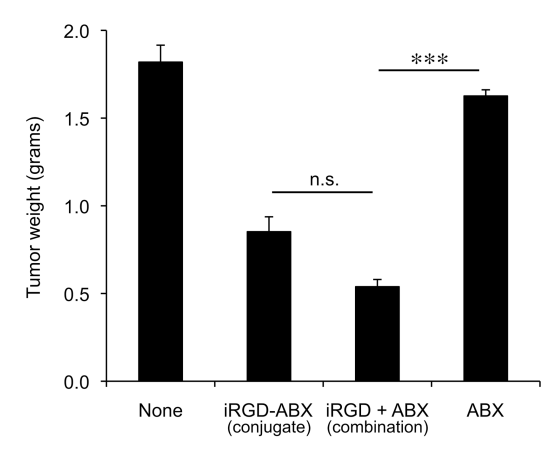

Fig. 1.

Comparison of the drug delivery efficiency of the iRGD combination regimen and conjugated drug delivery. (A and $\mathbf{C}$ ) Nab-paclitaxel (Abraxane; ABX) quantification in orthotopic human breast tumor (BT474)(A) and human prostate tumor (22Rv1)(C) xenograft models. ABX with free iRGD (combination), ABX coated with iRGD (conjugate), or ABX alone, was intravenously injected into tumor-bearing mice. Three hours later, $\mathrm{ABX}$ was captured from tumor extracts with an anti-taxol antibody, followed by detection with a human albumin antibody. $n=3$ per group. (B and $\mathbf{D})$ Long-term treatment of tumor mice with ABX. Mice bearing orthotopic BT474 (B) or 22Rv1 (D) tumors were intravenously injected with the indicated $\mathrm{ABX}$ formulations every other day at $3 \mathrm{mg}$ paclitaxel $/ \mathrm{kg} /$ injection, or with phosphate-buffered saline (PBS) only. The treatment was continued for 24 days in (B) and 16 days in (D). $n=8$ per group in (B) and $n=9$ in (D). Statistical analyses were performed with Student's $t$-test in (A) and (C) and ANOVA in (B) and (D). Error bars, mean \pm SEM; n.s., not significant; ${ }^{*} p<0.05 ; * * * p<0.001$. 
A

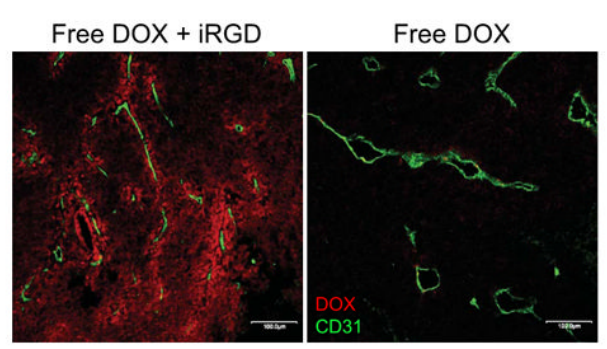

C

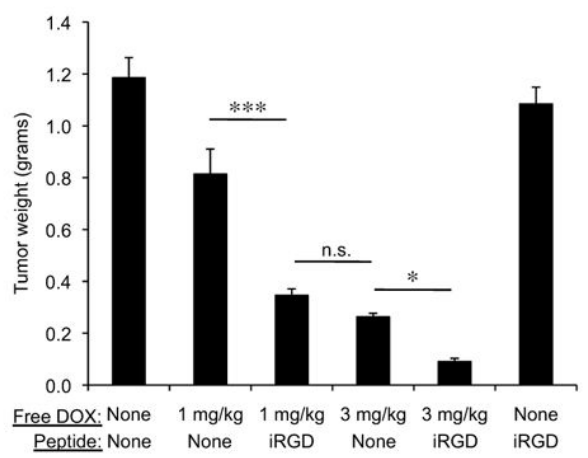

B

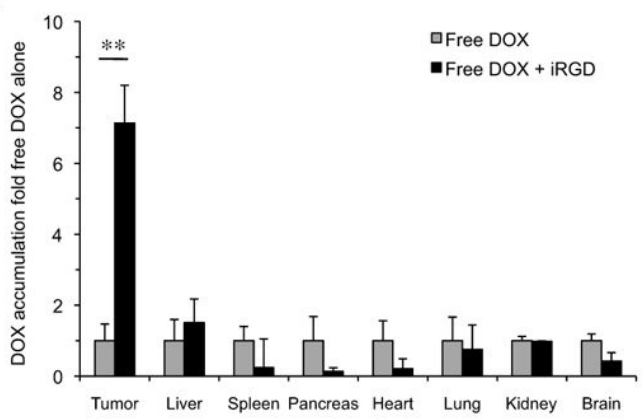

D

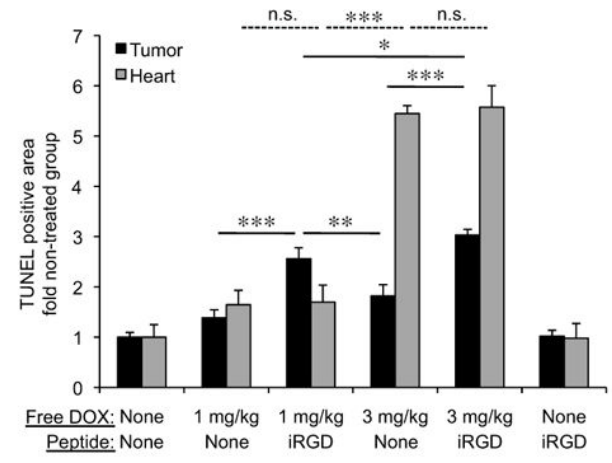

Fig. 2.

Enhanced anti-tumor effect of free DOX co-injected with iRGD. (A and B) Mice bearing orthotopic 22Rv1 human prostate tumors were intravenously injected with a mixture of DOX $(10 \mathrm{mg} / \mathrm{kg})$, and $4 \mu \mathrm{mol} / \mathrm{kg}$ of iRGD or PBS. Tumors and tissues were collected 1 hour later. $n=3$ per group. In (A), the tumors were sectioned and stained for blood vessels with an anti-CD31 and the native fluorescence was used to detect DOX. Scale bars $=100 \mu \mathrm{m}$. In (B), DOX in the tissues was quantified. (C) Mice bearing orthotopic 22Rv1 tumors implanted 2 weeks earlier received intravenous injections every other day of DOX ( 1 or 3 $\mathrm{mg} / \mathrm{kg}$ ) or PBS, combined with $4 \mu \mathrm{mol} / \mathrm{kg}$ of iRGD or PBS. The tumors were harvested and weighed after 24 days of treatment. $n=10$ per group. (D) TUNEL staining was performed on tumors and hearts from the treatment study, and quantified for positive staining.

Statistical analyses were performed with Student's $t$-test in (B), and ANOVA in (C) and (D); Error bars, mean \pm SEM; n.s., not significant; $* p<0.05$; ** $p<0.01 ; * * p<0.001$. 
A
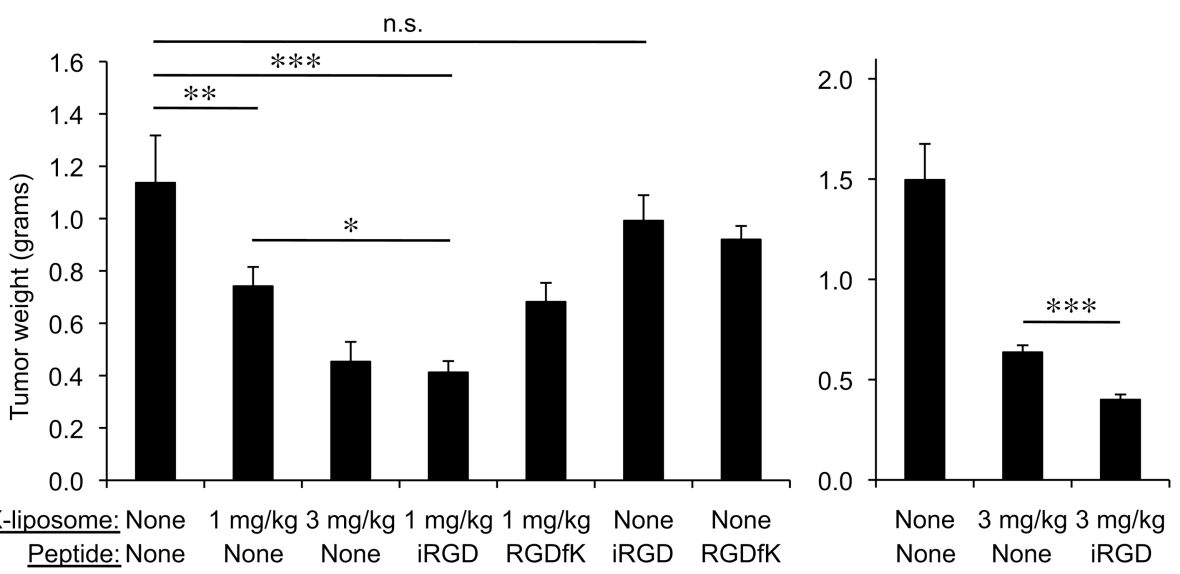

B

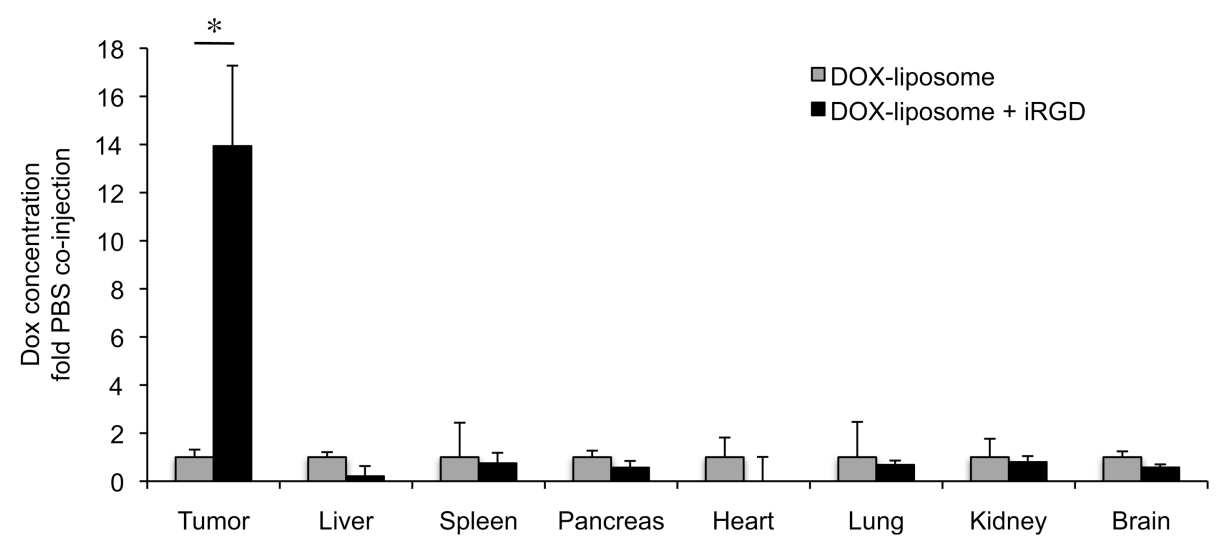

C

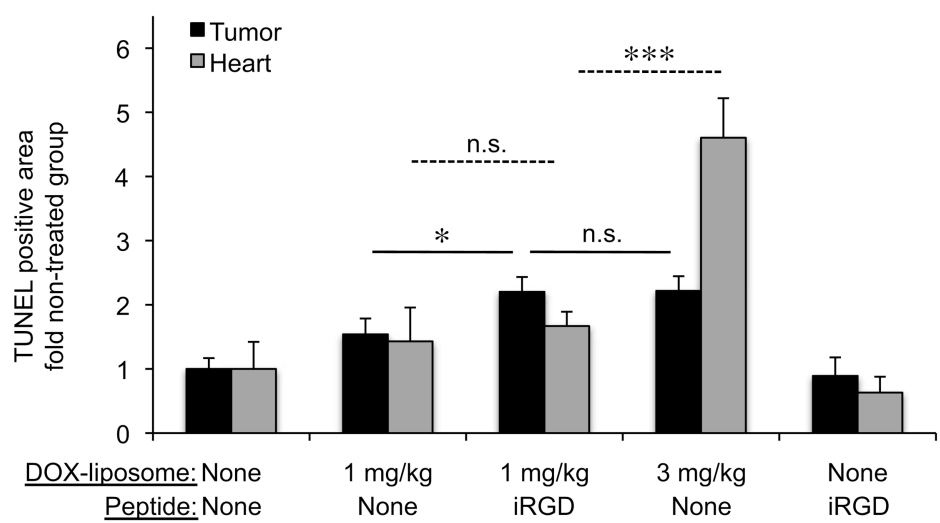

Fig. 3.

Enhanced anti-tumor effect of DOX-liposomes co-injected with iRGD. (A) Nude mice bearing orthotopic $22 \mathrm{Rv} 1$ human prostate tumors implanted 2 weeks earlier received daily intravenous injections of DOX-liposomes ( 1 or $3 \mathrm{mg} / \mathrm{kg}$ ) or PBS, combined with $2 \mu \mathrm{mol} / \mathrm{kg}$ of iRGD or cyclo(-RGDfK-), or PBS. The tumors were harvested and weighed after 17 days of treatment. $n=5$ per group in the left panel, and $n=8$ per group in the right panel. (B) Mice bearing orthotopic 22Rv1 tumors were intravenously injected with DOX-liposomes (5 $\mathrm{mg} / \mathrm{kg}$ ) followed by $4 \mu \mathrm{mol} / \mathrm{kg}$ of iRGD or PBS. The tumors and tissues were collected 3 hours later, and DOX in the tissues was quantified. $n=3$ per group. (C) TUNEL staining was performed on tumors and hearts from the treatment study in (A), and quantified for 
positive staining. Statistical analyses were performed with ANOVA in (A, left panel) and (C), and Student's $t$-test in (A, right panel) and (B). Error bars, mean \pm SEM; n.s., not significant; * $p<0.05 ; * * p<0.01 ; * * * p<0.001$. 
A

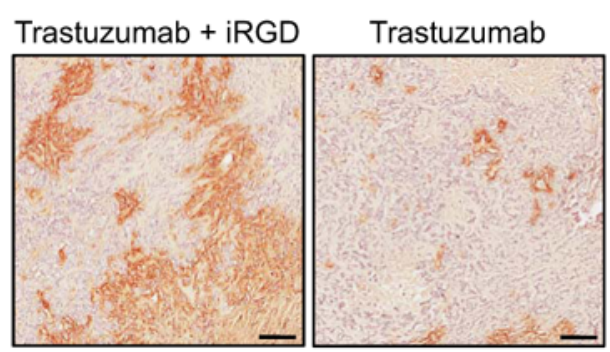

B

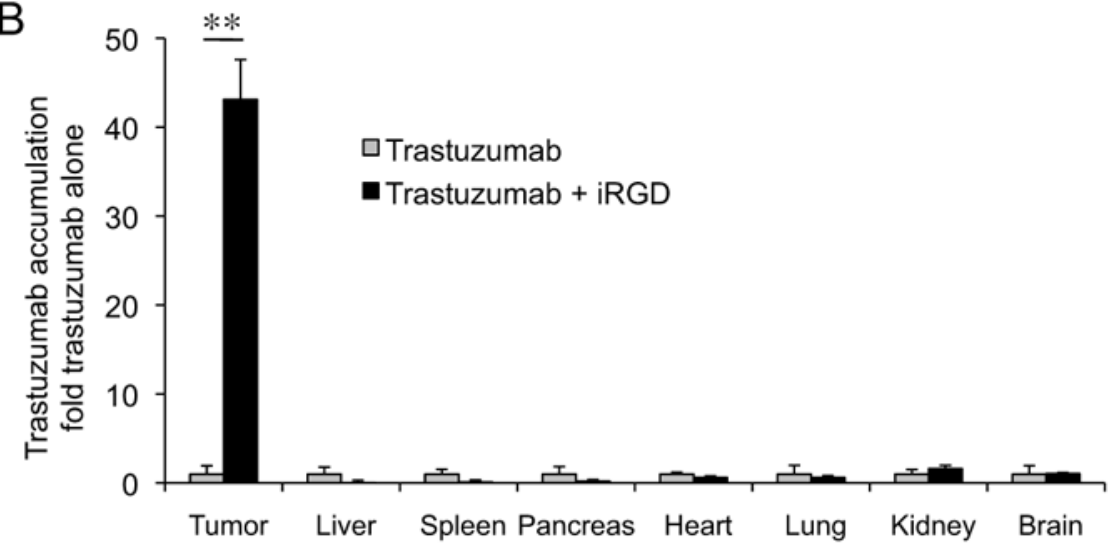

c

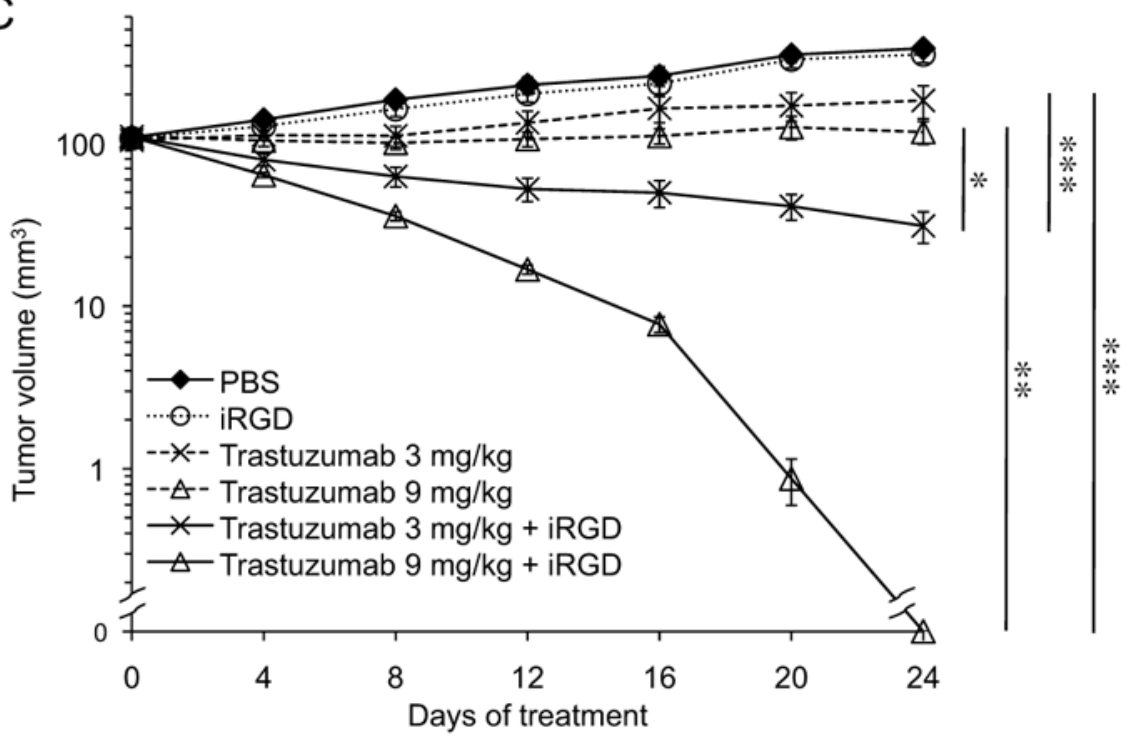

Fig. 4.

Enhanced anti-tumor effects of trastuzumab co-injected with iRGD. (A and B) Mice bearing orthotopic BT474 human breast tumors were intravenously injected with trastuzumab (3 mg/ $\mathrm{kg}$ ) followed by $4 \mu \mathrm{mol} / \mathrm{kg}$ of iRGD, or PBS. Tissues were collected 3 hours later. $n=3$ per group. In (A), tumor sections were stained for trastuzumab with an anti-human IgG antibody, and quantified for positive staining. Scale bars $=200 \mu \mathrm{m}$. In (B), trastuzumab in the tissues was quantified with a competitive ELISA. $n=3$ per group. (C) Tumor treatment study with coadministration of trastuzumab and iRGD. BT474 tumor mice were intravenously injected every 4 days with trastuzumab at 3 or $9 \mathrm{mg} / \mathrm{kg}$ on the first day of treatment (day 0) and 1.5 or $4.5 \mathrm{mg} / \mathrm{kg}$ in subsequent injections, or PBS. The treatment was 
combined with daily injections of $4 \mu \mathrm{mol} / \mathrm{kg}$ iRGD or PBS on the days of trastuzumab injection, and $2 \mu \mathrm{mol} / \mathrm{kg}$ iRGD or PBS on the other days. $n=10$ per group. One of 5 experiments that gave similar results is shown. Statistical analyses were performed with Student's $t$-test in (A) and (B), and ANOVA in (C). Error bars, mean \pm SEM; n.s., not significant; *p $<0.05 ; * * p<0.01 ; * * * p<0.001$. 